\title{
Dirac electrons in a Kronig-Penney potential: Dispersion relation and transmission periodic in the strength of the barriers
}

\author{
M. Barbier, ${ }^{1}$ P. Vasilopoulos, ${ }^{2}$ and F. M. Peeters ${ }^{1}$ \\ ${ }^{1}$ Department of Physics, University of Antwerp, Groenenborgerlaan 171, B-2020 Antwerpen, Belgium \\ ${ }^{2}$ Department of Physics, Concordia University, 7141 Sherbrooke Ouest, Montréal, Quebec, Canada H4B 1R6
}

(Received 23 October 2009; published 18 November 2009)

\begin{abstract}
The transmission $T$ and conductance $G$ through one or multiple one-dimensional, $\delta$-function barriers of two-dimensional fermions with a linear energy spectrum are studied. $T$ and $G$ are periodic functions of the strength $P$ of the $\delta$-function barrier $V(x, y) / \hbar v_{F}=P \delta(x)$. The dispersion relation of a Kronig-Penney (KP) model of a superlattice is also a periodic function of $P$ and causes collimation of an incident electron beam for $P=2 \pi n$ and $n$ integer. For a KP superlattice with alternating sign of the height of the barriers the Dirac point becomes a Dirac line for $P=(n+1 / 2) \pi$.
\end{abstract}

DOI: 10.1103/PhysRevB.80.205415

PACS number(s): 71.10.Pm, 73.21.-b, 81.05.Uw

\section{INTRODUCTION}

The study of particle motion in periodic potentials is at the heart of condensed-matter physics and it is usually assumed that the energy spectrum is parabolic. One of the earliest examples is the well-known, one-dimensional (1D) Kronig-Penney (KP) model $^{1}$ that consists of an infinite succession of very thin $(W \rightarrow 0)$ and very high $\left(V_{0} \rightarrow \infty\right)$ barriers, referred to as $\delta$-function barriers but such that their product $P \propto W V_{0}$ remains constant. This results in minibands in the electron spectrum.

One may wonder though how such results are modified if the energy is linear-in wave vector. Such a spectrum occurs for relativistic electrons with energy $E=\hbar c p \gg E_{0}=m_{0} c^{2}$, where $c$ is the speed of light and $m_{0}$ the bare electron rest mass. Even without neglecting $E_{0}$ a strict $1 \mathrm{D}$ Dirac KP model was considered for relativistic quarks. ${ }^{2}$ It is also known that electrons can transmit perfectly, upon normal incidence, through arbitrarily wide and high barriers, referred to as Klein paradox or Klein tunneling. ${ }^{3}$ With the discovery of graphene, ${ }^{4}$ a one-atom-thick layer of carbon atoms, another system became available in which particles (electrons) moving in two dimensions, have a linear spectrum, $E$ $=\hbar v_{F} k$, with $\vec{k}=\left(k_{x}, k_{y}\right)$ the wave vector. Importantly, carriers in graphene behave as chiral, massless fermions described by Dirac's equation without the mass term and move with the Fermi velocity $v_{F} \approx c / 300$. There is a wealth of exceptional properties of graphene; see, e.g., Ref. 5.

Because the carriers in graphene move in two dimensions, tunneling through barriers is inherently two dimensional (2D) and depends on the direction of the incident electron beam even in the absence of a magnetic field. Many authors, including ourselves, have studied this tunneling, through single, multiple barriers, and superlattices. ${ }^{6,7}$ Surprisingly, tunneling through $\delta$-function barriers has received very little attention $^{8}$ and we are not aware of any Dirac KP model for a superlattice in graphene. An interesting development was the application of periodic potentials to graphene that turned it into a self-collimating material despite the rather unusually high potentials used. ${ }^{9}$

Motivated by all these results and the absence of a systematic treatment of KP barriers or superlattices, we study in this work the transmission through such structures as well as the dispersion relation of a KP superlattice. Although the model may appear a bit unrealistic since a relatively smooth potential is needed to describe the carrier dynamics by the Dirac equation, ${ }^{10}$ its simplicity is attractive and elucidates certain symmetry properties of the spectrum. Furthermore one can realize the model by using a potential which is smooth on the scale of the atomic distance while the barrier width should remain small compared to the typical electron wavelength. The unexpected results mentioned in the abstract are in sharp contrast with those for carriers with a parabolic energy spectrum described by the Schrödinger equation. We will use graphene as an example but the results apply to any $2 \mathrm{D}$ system with a linear-in-wave-vector spectrum and a two-component spinor.

\section{TRANSMISSION THROUGH A $\delta$-FUNCTION BARRIER}

We describe the electronic structure of an infinitely large flat graphene flake in single valley approximation by the zero-mass Dirac equation and consider solutions with energy and wave vector near the $\mathrm{K}$ point. The Hamiltonian is $\mathcal{H}$ $=v_{F} \vec{\sigma} \cdot \vec{p}+1 V$ with $\vec{p}$ the momentum operator and 1 the 2 $\times 2$ unit matrix. In the presence of a $1 D$ potential $V(x)$ the equation $(\mathcal{H}-E) \psi=0$ admits solutions of the form $\psi(x) e^{i k_{y} y}$ where

$$
\psi(x)=\left(\begin{array}{c}
1 \\
s e^{i \phi}
\end{array}\right) e^{i \lambda x}, \quad \psi(x)=\left(\begin{array}{c}
1 \\
-s e^{-i \phi}
\end{array}\right) e^{-i \lambda x}
$$

with $\tan \phi=k_{y} / \lambda, s=\operatorname{sign}[\varepsilon-u(x)], \lambda=\left\{[\varepsilon-u(x)]^{2}-k_{y}^{2}\right\}^{1 / 2}, \varepsilon$ $=E / v_{F} \hbar$, and $u(x)=V(x) / v_{F} \hbar ; \varepsilon$ and $u(x)$ are in units of inverse length. As usual, we approximate a $\delta$-function barrier with a very thin and very high barrier, of width $W(\rightarrow 0)$ and height $V_{0}(\rightarrow \infty)$ but keep constant the dimensionless product $P=W V_{0} / \hbar v_{F}$ which we call its strength. Referring to Eq. (1) and Fig. 1, the wave function in each of the regions (1)-(3) can be written as a superposition of the eigenstates of Eq. (1) $\psi_{n}(x)=\mathcal{G}_{n} \mathcal{M}_{n}(x) \mathcal{A}_{n}, n=1,2,3$, with coefficients $\mathcal{A}=(A, B)^{T}$ and 

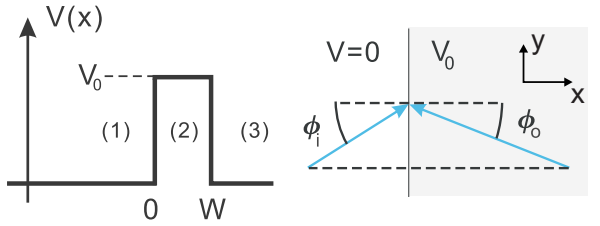

(a)

(b)

FIG. 1. (Color online) (a) 1D potential barrier of height $V_{0}$ and width $W$. (b) Wave-vector $\vec{k}=\left(k_{x}, k_{y}\right)$ geometry for an electron, with energy $0<E<V_{0}$, impinging on a square potential barrier (gray area).

$$
\mathcal{G}=\left(\begin{array}{cc}
1 & 1 \\
s e^{i \phi} & -s e^{-i \phi}
\end{array}\right), \quad \mathcal{M}(x)=\left(\begin{array}{cc}
e^{i \lambda x} & 0 \\
0 & e^{-i \lambda x}
\end{array}\right)
$$

Matching the wave function at the interfaces $x=0$ and $x=W$ gives the transfer matrix $\mathcal{N}=\mathcal{G}^{-1} \mathcal{S G}$ in terms of the matrix $S$ which relates the wave function in front of the barrier to the one after it in the manner $\psi_{1}(0)=S \psi_{3}(0+)$. The result is

$$
S=\mathcal{G}_{2} \mathcal{M}_{2}^{-1}(W) \mathcal{G}_{2}^{-1}=\left(\begin{array}{cc}
\cos P & i \sin P \\
i \sin P & \cos P
\end{array}\right) .
$$

We notice that $S$ is a periodic function of $P$ and that $S$ $= \pm 1$ for $P=n \pi$. This is a special situation in which the two pseudospin components of the wave function do not mix. Later on we will see that this periodicity appears in the transmission through a $\delta$-function barrier and in the dispersion relation of the KP model.

With the elements of $\mathcal{N}$ denoted by $n_{i j}$, the transmission is $T=|t|^{2}=1 /\left|n_{11}\right|^{2}$. The explicit result is

$$
T=1 /\left[1+\sin ^{2} P \tan ^{2} \phi\right],
$$

and coincides with the formula for transmission, found in Ref. 6, in the limit of $\delta$-function barriers. Obviously, $T$ and $R=1-T$ are periodic functions of $P$, that is, $X(P+n \pi, \phi)$ $=X(P, \phi)$ for $n$ integer and $X=T, R$. In addition, from Eq. (4) we find that $T(P, \phi)$ has the following properties:

(1) $T(P, \phi)=T(\pi-P, \phi)=T(\pi+P, \phi)$,

(2) $T(n \pi, \phi)=1$,

(3) $T(\pi / 2, \phi)=\cos ^{2} \phi$,

(4) $T(P, \phi=0)=1, \quad T \approx 1$ for $\phi \approx 0 \leftrightarrow k_{y} \ll k_{x}$,

(5) $T(P, \pm \pi / 2)=0, \quad T \approx 0$ for $\phi \approx \pm \pi / 2 \leftrightarrow k_{y} \gg k_{x}$.

These results are very different from those of the nonrelativistic case where $T$ is a decreasing function of $P$. A contour plot of the transmission is shown in Fig. 2(a). This figure shows clearly the symmetry properties $T(P, \phi)=T(P,-\phi)$ and $T(\pi-P, \phi)=T(P, \phi)$.

\section{Conductance}

The two-terminal conductance is $G$ $=G_{0} \int_{-\pi / 2}^{\pi / 2} T(P, \phi) \cos \phi d \phi$, with $G_{0}=2 E_{F} L_{y} e^{2} /\left(v_{F} h^{2}\right)$ and $L_{y}$
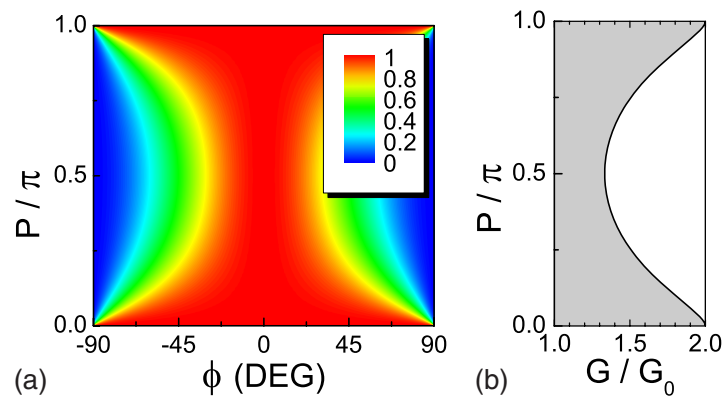

FIG. 2. (Color online) (a) Transmission $T$ through a $\delta$-function barrier vs its strength $P$ and the angle of incidence $\phi(\tan \phi$ $=k_{y} / k_{x}$ ). Only one period is shown. (b) Conductance $G / G_{0}$ vs $P$.

the width of the system. Using Eq. (4) for $T(P, \phi)$ the resulting $G$ is periodic in $P$ and given by

$$
G / G_{0}=2[1-\operatorname{arctanh}(\cos P) \sin P \tan P] / \cos ^{2} P .
$$

For one period, $G$ is shown in Fig. 2(b); its minimum value is $4 / 3$ and its maximum one 2 .

\section{TRANSMISSION THROUGH TWO $\delta$-FUNCTION BARRIERS}

We consider two barriers separated by a distance $L$ characterized by the potential $V(x, y) / \hbar v_{F}=P_{1} \delta(x)+P_{2} \delta(x-L)$, with strengths $P_{1,2}$ and introduce the dimensionless variables $\varepsilon \rightarrow \varepsilon L, k_{y} \rightarrow k_{y} L, u_{0} \rightarrow u_{0} L$, and $x \rightarrow x / L$. Due to space limitations we treat only the cases of parallel and antiparallel $\delta$-function barriers with the same strength $\left|P_{1}\right|=\left|P_{2}\right|$.

Parallel $\delta$-function barriers. This is a model system for a resonant tunneling structure ${ }^{6,7}$ and also for a Fabry-Perot interferometer whose resonances were recently investigated experimentally. ${ }^{11}$ The transmission is given by

$$
T=\left[1+\tan ^{2} \phi\left(\cos k_{x} \sin 2 P-2 s \sin k_{x} \sin ^{2} P / \cos \phi\right)^{2}\right]^{-1}
$$

with $s=\operatorname{sign}(\varepsilon)$. The properties of $T\left(P, \phi, k_{x}\right)$ are identical to those for a single barrier except for property (3) and property (1) which must be replaced by $T\left(P, \phi, k_{x}\right)=T\left(P+n \pi, \phi, k_{x}\right)$. In Fig. 3(a) we show $T\left(P, \phi, k_{x}\right)$ through two barriers with $P=\pi / 10$.


FIG. 3. (Color online) (a) Transmission through two parallel $\delta$-function barriers, as a function of the wave-vector component $k_{x}$ and angle of incidence $\phi$, for $P=\pi / 10$. The yellow solid curve shows the contour with a transmission value of 0.75 for $P=\pi / 2$. (b) As in (a) for two antiparallel $\delta$-function barriers. 

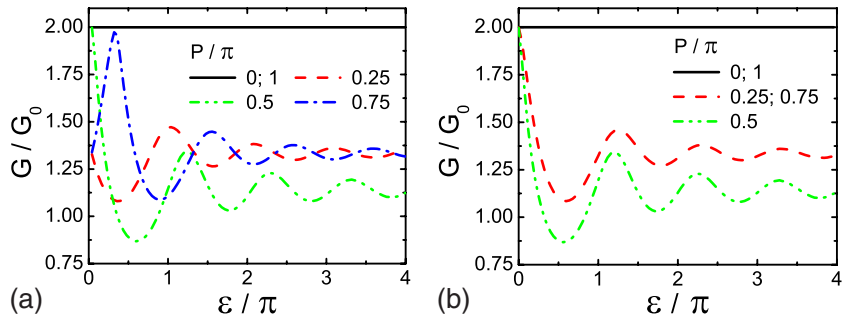

FIG. 4. (Color online) (a) Conductance $G\left(G_{0}\right)$ vs $\varepsilon$ for several strengths $P$ through two parallel $\delta$-function barriers. (b) As in (a) for two antiparallel barriers.

Antiparallel $\delta$-function barriers. We now consider two parallel $\delta$ functions but with opposite sign, $P=P_{1}=-P_{2}$. The result for the transmission is

$$
T=\left[\cos ^{2} k_{x}+\sin ^{2} k_{x}\left(1-\sin ^{2} \phi \cos 2 P\right)^{2} / \cos ^{4} \phi\right]^{-1} .
$$

In Fig. 3(b) we show the transmission through two opposite barriers for $P=\pi / 10$. The symmetry properties of $T\left(P, \phi, k_{x}\right)$ for a single barrier again hold, except for the value of $T\left(\pi / 2, \phi, k_{x}\right)$, see property (3). In addition, we now have $T\left(P, \phi, k_{x}\right)=T\left(P, \phi,-k_{x}\right)$.

The periodicity in the transmission is also present in the conductance $G$. We show $G$ in Fig. 4(a) for parallel and in Fig. 4(b) for antiparallel $\delta$-function barriers.

\section{KRONIG-PENNEY MODEL}

For an infinite number of periodic square barriers, one can tune the system into a self-collimating material. ${ }^{9}$ For special values of $V_{0}, W$, and $L$ it was found in Ref. 9 that the dispersion relation near the $\mathrm{K}$ point is almost linear in $k_{x}$ and constant along $k_{y}$. The system thus behaves as a 1D metal. We look for similar results using $\delta$-function barriers. Within the KP model we replace the square barriers by $\delta$-function barriers, characterized by $V(x, y) / \hbar v_{F}=\sum_{j=-\infty}^{\infty} P \delta(x-j L)$. The resulting wave function is a Bloch function and the transfer matrix $\mathcal{N}$ pertinent to these barriers leads to $\psi(1)=e^{i k_{x}} \psi(0)$ and $\mathcal{A}_{1}=\mathcal{N} \mathcal{A}_{2}$, with $k_{x}$ the Bloch wave vector. From these conditions we can extract the relation $e^{-i k_{x}} \mathcal{M}(1) \mathcal{A}_{2}=\mathcal{N} \mathcal{A}_{2}$, with $\mathcal{M}(x)$ given by Eq. (2). Then setting the determinant of the coefficients in $\mathcal{A}_{2}=(A, B)^{T}$ equal to zero and using the transfer matrix for a $\delta$-function barrier leads to $\left(\lambda=\left[\varepsilon^{2}\right.\right.$ $\left.\left.-k_{y}^{2}\right]^{1 / 2}\right)$,

$$
\cos k_{x}=\cos P \cos \lambda+(\varepsilon / \lambda) \sin \lambda \sin P .
$$

The solution of Eq. (9) gives the dispersion which is periodic in $P$ and the spectrum is shown in Fig. 5 for $P=\pi / 2$. Further, Eq. (9) is mapped, for $k_{y}=0$, directly onto that for strictly $1 \mathrm{D}$ fermions ${ }^{2}$ and gives the spectrum

$$
\varepsilon=P \pm k_{x}+2 n \pi
$$

with $n$ an integer.

Equation (9) contrasts very sharply with that for 2D electrons with a parabolic spectrum in a 1D KP potential which, with $\lambda^{\prime}=\left[2 \mu \varepsilon-k_{y}^{2}\right]^{1 / 2}$ and $\mu=m v_{F} L / \hbar$, reads

$$
\cos k_{x}=\cos \lambda^{\prime}+\left(\mu P / \lambda^{\prime}\right) \sin \lambda^{\prime},
$$

the resulting dispersion relation is not periodic in $P$.
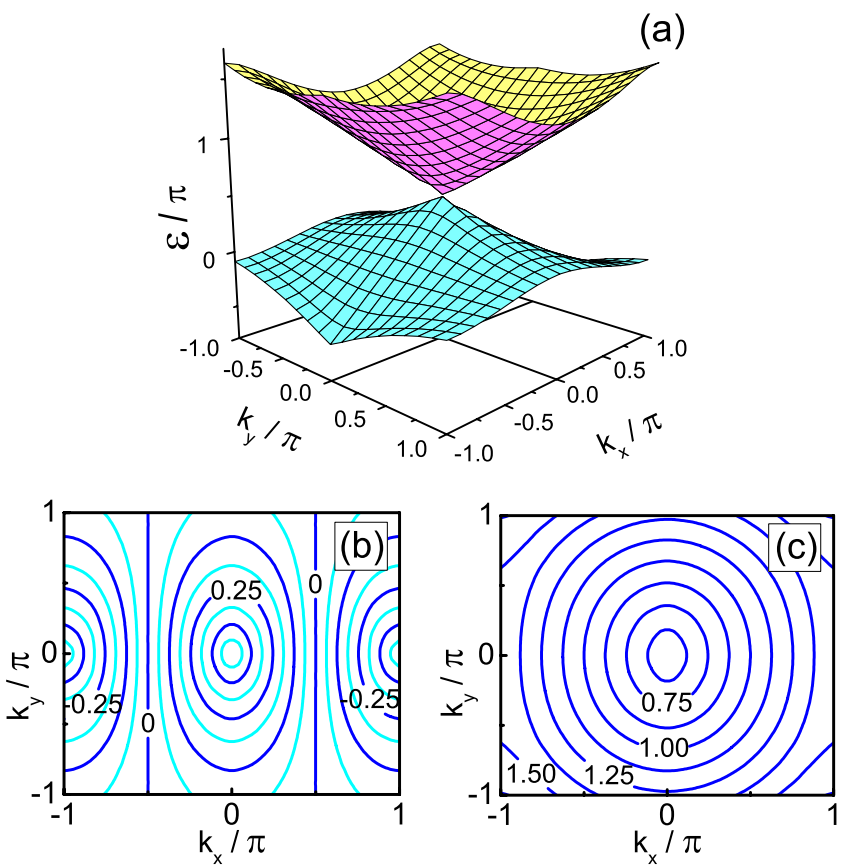

FIG. 5. (Color online) (a) Energy bands, near the Fermi level, close to the $\mathrm{K}$ point in the KP model, for $P=\pi / 2$. The valence band touches the conduction band at $\varepsilon=P=\pi / 2$. For large $k_{y}$ the valence band becomes flat. (b) and (c) projections, respectively, of the valence and conduction bands onto the $\left(k_{x}, k_{y}\right)$ plane.

\section{Properties of the spectrum}

Since the dispersion relation is periodic in $P$, with period $2 \pi$, it is sufficient to study it only for $0 \leq P \leq 2 \pi$. For particular values of $P$ we find

$$
\begin{aligned}
& \text { (1) } P=2 \pi \rightarrow \varepsilon= \pm\left[k_{y}^{2}+\left(k_{x}+2 n \pi\right)^{2}\right]^{1 / 2}, \\
& \text { (2) } P=\pi \rightarrow \varepsilon= \pm\left\{k_{y}^{2}+\left[k_{x}+(2 n+1) \pi\right]^{2}\right\}^{1 / 2}, \\
& \text { (3) } P=\pi / 2(3 \pi / 2) \rightarrow \cos k_{x}=+(-) \varepsilon \sin \lambda / \lambda .
\end{aligned}
$$

In limiting cases we are able to obtain explicit expressions for $E=E\left(k_{x}, k_{y}\right)$. We expand the dispersion relation for small $k_{y}$ and $\varepsilon-P$. The resulting quadratic equation for $\varepsilon$ is solved by

$$
\varepsilon_{ \pm} \approx P \pm\left[4 \sin ^{2}\left(k_{x} / 2\right)+\left(k_{y}^{2} / P^{2}\right) \sin ^{2} P\right]^{1 / 2} .
$$

For small $k_{x}$ we can replace the term $4 \sin ^{2}\left(k_{x} / 2\right)$ by $k_{x}^{2}$. Notice that for $k_{x}=0$ we find $\varepsilon_{ \pm} \approx P \pm k_{y} \sin P / P$ which is a linear spectrum with a reduced velocity. For $k_{y}=0$, we have $\varepsilon_{+}=P \pm 2 \sin \left(\left|k_{x}\right| / 2\right)$, which is linear for small $k_{x}$ but possesses a typical band shape for large $k_{x} \approx \pi$. For small $k_{x}$ $>k_{y} \sin P / P$ we have

$$
\varepsilon_{ \pm} \approx P \pm\left(2 k_{x}^{2}+k_{y}^{2} \sin ^{2} P / P^{2}\right) / 2\left|k_{x}\right| .
$$

For $P \gg 1, \varepsilon$ is highly anisotropic and nearly flat vs $k_{y}$.

Relation to the spectrum of a square superlattice. We now look whether we can find an energy spectrum similar to that of Ref. 9 pertinent to square barriers, with height $V_{0}$ $=720 \mathrm{meV}$, width $W=5 \mathrm{~nm}$, and unit-cell length $L$ $=10 \mathrm{~nm}$. In our units these values correspond to $P$ 

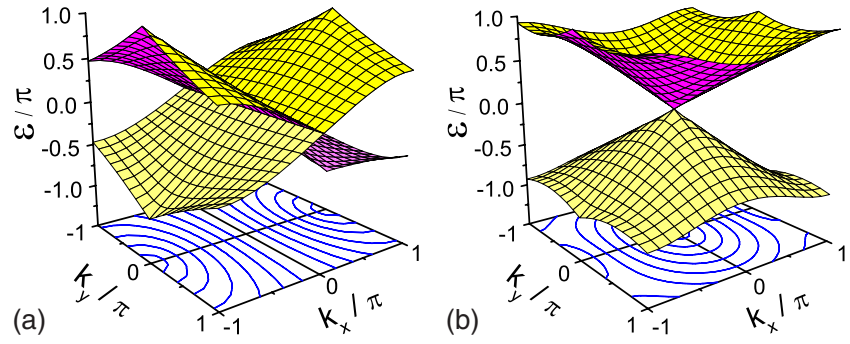

FIG. 6. (Color online) (a) Conduction and valence bands, near the $\mathrm{K}$ point, in the extended KP model, for $P=\pi / 2$. The bands form a "cross" in the $\left(E, k_{x}\right)$ plane and the Dirac point has become a Dirac line. The dispersion is nearly independent of $k_{y}$. (b) As in (a) for $P=\pi / 4$.

$=V_{0} W / v_{F} \hbar=2 \pi$ and lead to $\varepsilon= \pm\left[k_{y}^{2}+\left(k_{x}+2 n \pi\right)^{2}\right]^{1 / 2}$. Since the Fermi level in these units is $\varepsilon_{F}=2 \pi=P$, we look for the spectrum near the value $\varepsilon=\left[k_{y}^{2}+\left( \pm\left|k_{x}\right|+2 \pi\right)^{2}\right]^{1 / 2}$. Although these bands seem to fulfill our demands because the dispersion looks rather flat in the $k_{y}$ direction, the concern is that we would obtain the same dispersion for $P \rightarrow 0$ and $\varepsilon_{F}$ $\rightarrow 2 \pi$. But this can be obtained by folding the conelike dispersion of graphene and results simply from working in the reduced-zone scheme. Consequently no new fundamental physics should be attached to it. Further, from this correspondence we expect and found that for square barriers with $P$ $=2 \pi n$, the situation is more favorable for the occurrence of collimation. It follows that the collimation effect is also obtainable for barriers that are lower than the unusually high ones of Ref. 9 if one uses longer unit-cell periods.

\section{EXTENDED KRONIG-PENNEY MODEL}

The square barriers are replaced by alternating-in-sign $\delta$-function barriers. The unit cell of the periodic potential contains one such barrier up, at $x=0$, followed by a barrier down, at $x=1 / 2$. The resulting transfer matrix leads to

$$
\cos k_{x}=\cos \lambda-\left(2 k_{y}^{2} / \lambda^{2}\right) \sin ^{2}(\lambda / 2) \sin ^{2} P,
$$

where $\tan \phi=k_{y} / \lambda$. From Eq. (15) we deduce that the dispersion is periodic in $P$, with period $\pi$, and has the following properties:

(1) it is invariant for $\varepsilon \rightarrow-\varepsilon$ and $P \rightarrow \pi-P$,

(2) $P=n \pi \rightarrow \varepsilon= \pm\left[k_{y}^{2}+\left(k_{x}+2 n \pi\right)^{2}\right]^{1 / 2}$,

(3) $P=\pi / 2 \rightarrow\left(\varepsilon, k_{x}, k_{y}\right)=\left(0,0, k_{y}\right)$.

In Fig. 6(a) we show the spectrum for $P=\pi / 2$. As seen, it is almost independent of $k_{y}$ for small energies while in the $k_{x}$ direction the bands are linear; this is an advantageous situation for self-collimation. For $k_{y}=0$ we obtain the linear spectrum

$$
\varepsilon= \pm\left|k_{x}\right|+2 n \pi,
$$

with the Dirac point at $\varepsilon=0$. We can also find an explicit expression for $k_{x} \approx 0$. Solving Eq. (15) gives

$$
\varepsilon_{ \pm}= \pm\left|k_{y} \cos P\right| \text {. }
$$

Then the group velocity $v_{y} \propto \partial \varepsilon / \partial k_{y}$ becomes small if $P$ $\approx \pi / 2+n \pi$. Figure 6(b) shows the energy spectrum for $P$ $=\pi / 4$, the Dirac cone becomes anisotropic, as the spectrum flattens in the $k_{y}$ direction.

We now consider the case where $k_{x}$ and $k_{y}$ are nonzero. If $\varepsilon \ll 1$ then the right-hand side of Eq. (15) can be expanded in $\varepsilon$. This leads to a quadratic equation for $\varepsilon$ with solutions

$$
\begin{aligned}
\varepsilon \approx & \pm\left|k_{y}\right|\left[\frac{\cosh k_{y}-\cos k_{x}-f}{\left(k_{y} / 2\right) \cos ^{2} P \sinh k_{y}+f}\right]^{1 / 2} \stackrel{P=\pi / 2}{\longrightarrow} \\
& \pm\left|k_{y}\right| \sin \left(\left|k_{x}\right| / 2\right) / \sinh \left(\left|k_{y}\right| / 2\right),
\end{aligned}
$$

where $f=2 \sin ^{2} P \sinh ^{2}\left(k_{y} / 2\right)$. For $k_{y}=0$ we find the result given by Eq. (13), that is, $\varepsilon_{ \pm}= \pm 2 \sin \left(\left|k_{x}\right| / 2\right)$ which is linear for small $k_{x}$.

\section{CONCLUSIONS}

In summary, we studied the transmission and conductance of fermions, with energy linear-in wave vector, through one and two $\delta$-function barriers and the energy spectrum of a KP superlattice. For very high $\left(V_{0} \rightarrow \infty\right)$ and very thin $(W \rightarrow 0)$ barriers we showed that they are periodic functions of their strength $P=W V_{0} / \hbar v_{F}$, where $v_{F}$ is the Fermi velocity. Further, we showed that a KP superlattice has an energy spectrum that is a periodic function of $P$, which is in sharp contrast with that obtained from the Schrödinger equation. An important consequence of that is collimation of an incident electron beam ${ }^{9}$ that here occurs for $P=2 \pi n$ with $n$ an integer. We also obtained various explicit but approximate dispersion relations, e.g., for small wave vector $\vec{k}=\left(k_{x}, k_{y}\right)$. Given the intense research activity in graphene and the very recent experimental verification of Klein tunneling, ${ }^{12}$ we expect that this periodic dependence on the strength $P$ will be tested in a near future.

\section{ACKNOWLEDGMENTS}

This work was supported by IMEC, the Flemish Science Foundation (FWO-Vl), the Belgian Science Policy (IAP), and the Canadian NSERC under Grant No. OGP0121756.
${ }^{1}$ C. Kittel, Introduction to Solid State Physics, 5th ed. (John Wiley \& Sons, New York, 1976).

${ }^{2}$ B. H. J. McKellar and G. J. Stephenson, Jr., Phys. Rev. C 35, 2262 (1987)
${ }^{3}$ O. Klein, Z. Phys. 53, 157 (1929).

${ }^{4}$ K. S. Novoselov, A. K. Geim, S. V. Morozov, D. Jiang, Y. Zhang, S. V. Dubonos, I. V. Grigorieva, and A. A. Firsov, Science 306, 666 (2004). 
${ }^{5}$ A. H. Castro Neto, F. Guinea, N. M. R. Peres, K. S. Novoselov, and A. K. Geim, Rev. Mod. Phys. 81, 109 (2009); C. W. J. Beenakker, ibid. 80, 1337 (2008).

${ }^{6}$ M. I. Katsnelson, K. S. Novoselov, and A. K. Geim, Nat. Phys. 2, 620 (2006); A. De Martino, L. Dell'Anna, and R. Egger, Phys. Rev. Lett. 98, 066802 (2007); Vitor M. Pereira and A. H. Castro Neto, ibid. 103, 046801 (2009).

${ }^{7}$ J. M. Pereira, Jr., P. Vasilopoulos, and F. M. Peeters, Appl. Phys. Lett. 90, 132122 (2007); M. Barbier, F. M. Peeters, P. Vasilopoulos, and J. M. Pereira, Jr., Phys. Rev. B 77, 115446 (2008); M. Ramezani Masir, P. Vasilopoulos, and F. M. Peeters, Appl.
Phys. Lett. 93, 242103 (2008).

${ }^{8}$ S. Ghosh and M. Sharma, J. Phys.: Condens. Matter 21, 292204 (2009).

${ }^{9}$ C.-H. Park, Y.-W. Son, L. Yang, M. L. Cohen, and S. G. Louie, Nano Lett. 8, 2920 (2008).

${ }^{10}$ N. H. Shon and T. Ando, J. Phys. Soc. Jpn. 67, 2421 (1998).

${ }^{11}$ S. Cho and M. S. Fuhrer, arXiv:0901.4157 (unpublished).

${ }^{12}$ N. Stander, B. Huard, and D. Goldhaber-Gordon, Phys. Rev. Lett. 102, 026807 (2009); A. F. Young and P. Kim, Nat. Phys. 5, 222 (2009). 